\title{
Anaphylaxis to Cisplatin after Carboplatin Hypersensitivity Reaction in Advanced Non-Small Cell Lung Cancer
}

\author{
Chau Thi Ngoc Dieu1, Gia Hoang Nguyen², Quynh Thi Hong Mai ${ }^{3}$ \\ ${ }^{1}$ Department of Pharmacy, Hanoi Oncology Hospital, Hanoi, Vietnam \\ ${ }^{2}$ Medical Oncology I Department, Hanoi Oncology Hospital, Hanoi, Vietnam \\ ${ }^{3}$ On-Demand General Medical Oncology Department, Hanoi Oncology Hospital, Hanoi, Vietnam \\ Email:chaudtn@bvubhn.vn, gianh@bvubhn.vn,quynhmth@bvubhn.vn
}

How to cite this paper: Dieu, C.T.N., Nguyen, G.H. and Mai, Q.T.H. (2021) Anaphylaxis to Cisplatin after Carboplatin Hypersensitivity Reaction in Advanced Non-Small Cell Lung Cancer. Case Reports in Clinical Medicine, 10, 295-302.

https://doi.org/10.4236/crcm.2021.1010037

Received: September 19, 2021

Accepted: October 17, 2021

Published: October 20, 2021

Copyright $\odot 2021$ by author(s) and Scientific Research Publishing Inc. This work is licensed under the Creative Commons Attribution International License (CC BY 4.0).

http://creativecommons.org/licenses/by/4.0/

\begin{abstract}
Patients with non-small cell lung cancer (NSCLC) who have received more than one cycle of platinum-based chemotherapy in their lifetime may be at risk of hypersensitivity. The overall incidence of hypersensitivity to carboplatin ranges from $1 \%$ to $27 \%$ and that of hypersensitivity to carboplatin is between $5 \%$ and $20 \%$. However, the actual incidence of cross-reactivity between platinum salts has not yet been known. In this paper, we reported a case of a 39-year-old man with advanced non-small cell lung cancer, who had cisplatin anaphylaxis after having had carboplatin hypersensitivity at the previous cycle. The anaphylactic reaction was managed successfully with adrenaline, corticosteroids, antihistamines, oxygen mask and isotonic fluid support. No further reactions were observed and after that he stopped the platinum regimen. This case illustrates that NSCLC patients may experience hypersensitive and cross-reactivity to both carboplatin and cisplatin. It is important to be aware of the possibility of anaphylaxis so that appropriate premedication or effective treatment can be promptly instituted.
\end{abstract}

\section{Keywords}

Non-Small Cell Lung Cancer, Anaphylaxis, Cisplatin, Carboplatin, Hypersensitivity

\section{Introduction}

Worldwide, lung cancer remains the leading cause of cancer death, with an estimated 1.8 million deaths (18\%) in 2020 [1]. Although the disease can be alleviated with comprehensive treatment, recurrence still appears in most patients 
with advanced-stage disease. Recently, targeted therapy and immunotherapy have changed the treatment landscape of non-small cell lung cancer (NSLCL). However, platinum-based chemotherapy is still the standard treatment for most patients with recurrent or advanced NSCLC [2].

Carboplatin and cisplatin have been shown to be effective chemotherapeutics and can be used to treat many solid tumors. Both carboplatin and cisplatin are platinum complexes, exerting their anticancer properties via the formation of DNA adducts that inhibit the replication and cause apoptosis [3]. The widespread use of platinum compounds in chemotherapy has led to the significantly increased incidence of hypersensitivity reactions [4]. Generally, hypersensitive reactions to carboplatin occur only after the patient has undergone several treatment cycles. In the first five cycles, the overall risk is less than 1\% [5]; it increases sharply to $6.5 \%$ by the sixth cycle and has been reported up to $27 \%$ in patients having received more than seven cycles [6]. The rate of hypersensitivity to cisplatin is similar to that of carboplatin. It is between $5 \%$ and $20 \%$ and increases with concomitant radiation [7]. Symptoms of platinum hypersensitivity can appear acutely during the infusion or within a few minutes, hours, or days after the infusion [8]. Common symptoms include rash, flushing, abdominal cramps, itchiness and back pain [4]. Severe anaphylaxis is rarely observed, mainly reported in individual case reports, with the majority of cases are among ovarian cancer patients [9] [10], while very few cases are in lung cancer patients. Here, with the patient's consent, we report a case of hypersensitivity and cross-reactivity to cisplatin and carboplatin in a patient with stage IIIb NSCLC. Through presenting this case report, we hope to raise doctors' and nurses' awareness on the possibility of platinum hypersensitivity in the lung cancer patients.

\section{Case Presentation}

The patient is a 39-year-old male with a past medical history of stage IIIB (T3N2M0) NSCLC (adenocarcinoma), who was treated with left lower lobe lobectomy with R2 resection in March 2016. FISH analysis identified that he has an ALK rearrangement.

He was received concurrent chemo-radiation including pemetrexed $\left(500 \mathrm{mg} / \mathrm{m}^{2}\right)$, carboplatin (AUC5) given in 4 cycles, and chest irradiation (60 Gy) at the time of diagnosis. In June 2019, the patient's local recurrence was found with a left lung lesion measuring 3 centimeters in diameter. He then was treated with crizotinib for 2 cycles, and afterward the disease has progressed. He had no reported history of food or medication allergies.

In December 2019, the patient was hospitalized with cough, breath shortness and tightness of chest. Chest CT revealed: a centrally located tumor with the dimension of $12 \times 50 \mathrm{~mm}$ having invasive adhesion in hilum and pleura (mostly mediastinal pleura) (as shown in Figure 1); there were some well-defined pulmonary nodules sized $3-5 \mathrm{~mm}$ in the rest of lung parenchyma, and a small volume of pleural fluid, about $10 \mathrm{~mm}$ in thickness; lower jugular nodes and left 


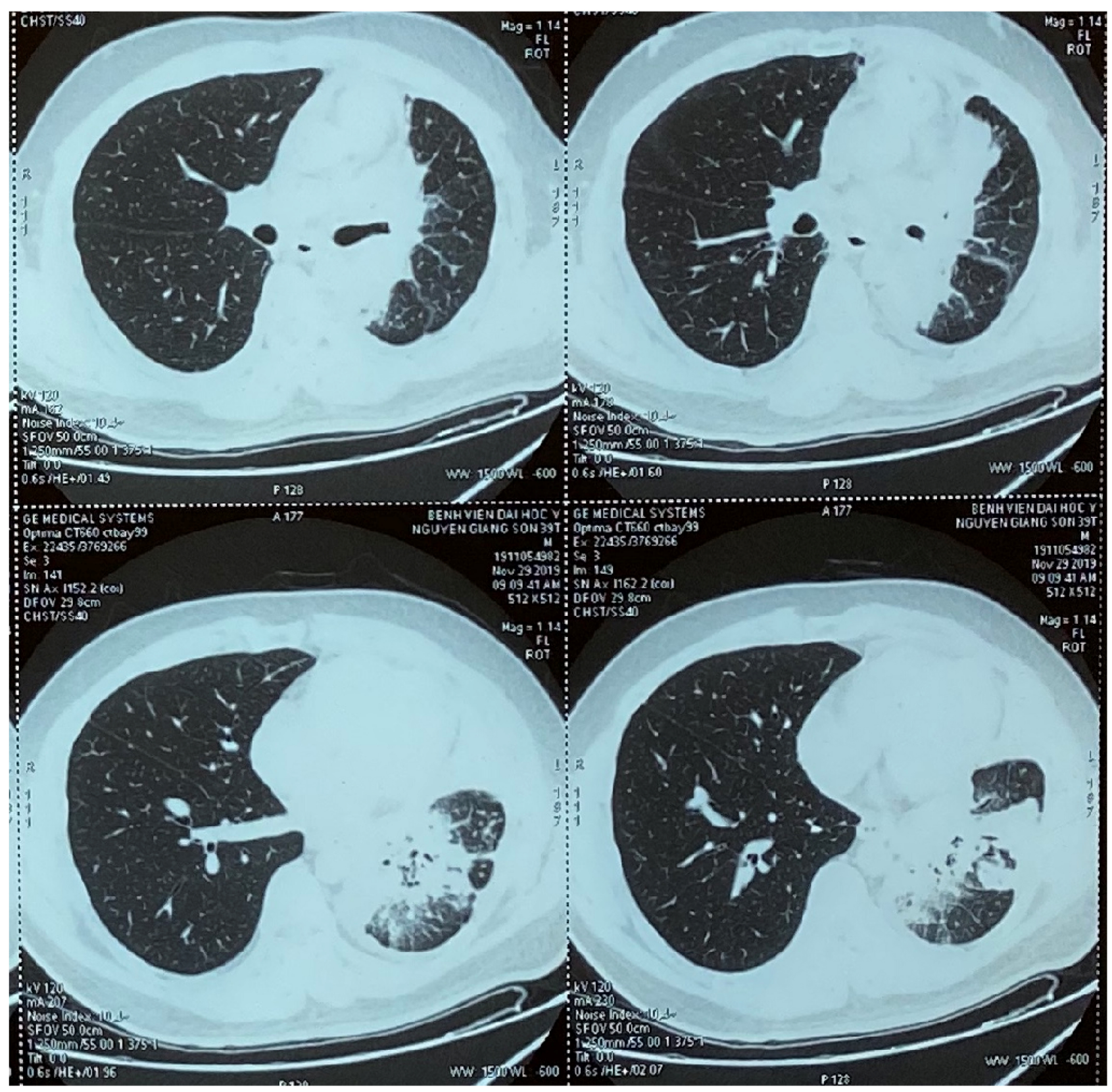

Figure 1. Chest CT result in Dec 2019.

supraclavicular lymph nodes were $6 \mathrm{~mm}$ in diameter. Periaortic lymph nodes were $8 \mathrm{~mm}$ in diameter.

The levels of the significant serum tumor markers, including carcinoembryonic antigen and circulating cytokeratin 19 fragments (CYFRA21-1) were 4.2 $\mathrm{ng} / \mathrm{mL}$ and $3.6 \mathrm{ng} / \mathrm{mL}$, respectively. The patient was clinically diagnosed with recurrent lung cancer, pleural metastases and supraclavicular metastases.

He then began treatment with intravenous carboplatin (AUC5) and pemetrexed $\left(500 \mathrm{mg} / \mathrm{m}^{2}\right)$, the cycle of chemotherapy was 21 days. He had partial response after 2 cycles of treatment.

During the third cycle of this chemotherapy regimen, he received pemetrexed 
for over 20 minutes, and then, 30 minutes later, carboplatin. Five minutes after starting the carboplatin infusion (his seventh lifetime exposure to carboplatin), the patient reported warm feeling in his face, itchy back and hands, nausea and vomit, followed by chest pressure, breathing difficulty and anxiety. Vital signs at the time of symptom onset were:

- Temperature: 36.5 degrees C.

- Blood pressure: 80/40 mmHg.

- Pulse: $44 \mathrm{bpm}$.

- Pulse oxygenation: $84 \%$ in room air.

Intervention: The carboplatin infusion was stopped. We initiated treatment immediately with $0.5 \mathrm{ml}$ of adrenaline $1 \mathrm{mg} / \mathrm{ml}$ (1:1000), hydration (normal saline $1 \mathrm{~L}$ in 1 hour), oxygen mask $5 \mathrm{~L} / \mathrm{min}$, diphenhydramine $25 \mathrm{mg}$ and methylprednisolone $40 \mathrm{mg}$.

His symptoms had been ultimately resolved without further intervention, and vital signs recorded 20 minutes after intravenous steroid administration were stable with blood pressure of 103/74 mmHg, oxygen saturation of $91 \%$ and pulse rate of $110 \mathrm{bpm}$. Carboplatin had been removed from further cycles of chemotherapy.

In the next (4th) cycle, carboplatin was replaced by cisplatin $75 \mathrm{mg} / \mathrm{m}^{2}$. Antihistamines and steroids were injected 1 hour before administration of cisplatin. However, he experienced severe hypersensitivity reaction again. Five minutes after cisplatin infusion, he suddenly had swollen tongue and face, sweating, abdominal cramps, nausea, shortness of breath and dizziness. His oral temperature was 36.3 degrees C, pulse rate was $70 \mathrm{bpm}$ and blood pressure was $69 / 46 \mathrm{mmHg}$. The initial oxygen saturation was $80 \%$ (in room air).

The immediate intervention was implemented as following:

- The cisplatin infusion was stopped.

- Adrenaline $1 \mathrm{mg} / 1 \mathrm{ml}(0.5 \mathrm{ml})$ was injected intramuscularly twice at 5 -minute intervals.

- Normal saline (1 L) was rapidly administered, plus, the patient received intravenous diphenhydramine $25 \mathrm{mg}$, methylprednisolone $80 \mathrm{mg}$ and oxygen mask $7 \mathrm{~L} / \mathrm{min}$.

- Adrenaline $1 \mathrm{mg} / 1 \mathrm{ml}(0.5 \mathrm{ml})$ was injected intramuscularly twice at 5-minute interval.

After 40 minutes, $\mathrm{BP}$ and $\mathrm{SpO}_{2}$ increased to $113 / 70 \mathrm{mmHg}$ and $97 \%$, respectively, his symptoms subsided gradually and no further reactions were observed.

The patient was followed up closely for 24 hours after the onset of symptoms and then discharged with stable vital signs. After that, he stopped the platinum regimen and received ceritinib $450 \mathrm{mg}$ per day. We continue measuring his CEA and monitoring his treatment response through chest CT scan and brain MRI every 3 months. At present (September 2021), the patient's overall status is stable: vital signs within normal limit, ECOG-PS score of 0 , no cough, no dyspnea andhaving partial response to ceritinib. 


\section{Discussion}

Delayed hypersensitive reactions to carboplatin are relatively common in patients who have previously received those drugs. In a study conducted by Morgan et al., among 34 patients with relapsed ovarian cancer who were given second and third cycles of carboplatin, as many as 15 (44\%) of them, developed postrelapsed hypersensitivity [11]. Sugimoto et al. reported that patients with carboplatin-free interval $>13$ months had a 22 -fold increased risk of hypersensitivity and that a maximum dose/body of carboplatin $>650 \mathrm{mg}$ is also an independent risk factor [12].

Our patient had received totally 7 cycles of carboplatin during the course of his treatment. He was given additional medication after a long platinum-free interval of more than 3 years that might increase the risk. The patient' symptoms developed very quickly, within a few minutes after starting carboplatin infusion. The initial symptoms were itchy back and hands, nausea, followed by respiratory symptoms and hypotension. These symptoms were largely consistent with type 1 hypersensitivity reaction, an IgE-mediated hypersensitivity that associates with repeated exposure to the agent. IgE bound to tissue mast cells and basophils in peripheral blood, causing the release of histamines, leukotrienes and prostaglandins, leading to telangiectasia and rapid smooth muscle contraction. This causes rash, flushing, and itchiness [13]. More severe reactions are bronchospasm, tachycardia, hypotension or hypertension, seizures, and chest pain. In a study on 69 patient with relapsed ovarian cancer, up to $46.7 \%$ of patients experienced hypersensitivity reactions with serious cardiovascular or respiratory events [14].

Hypersensitivity to cisplatin has also been observed in our patient reported here. Successful replacement of carboplatin by cisplatin has been reported in women with gynecological cancer but the actual frequency of cross-reactivity between them has not yet been known [15] [16]. Ottaiano et al. reported that ten consecutive patients with recurrent ovarian carcinoma, presenting with moderate or severe hypersensitivity reactions to carboplatin treated with cisplatin 60 $\mathrm{mg} / \mathrm{m}^{2}$ were generally well tolerated. A mild allergic reaction was recorded in only one case, after one cycle of cisplatin, and the patient was not re-exposed due to disease progression [15]. Intradermal skin test can be a useful tool to detect a possible cross-reaction between platinum salts. Syrigo et al. performed intradermal testing with a series of dilutions of carboplatin and cisplatin in three patients who had mild and severe hypersensitivity reactions to carboplatin. The result with carboplatin was positive in all patients and all of them were negative with cisplatin. The administration of cisplatin instead of carboplatin was well tolerated in all patients without the need of premedication [16]. Therefore, even though our patient had a carboplatin allergy, he still received cisplatin as an alternative and pre-prophylactic with corticosteroids and antihistamines. However, cases of fatal cisplatin reaction after carboplatin hypersensitivity have been reported. Dizon et al. reported one patient with carboplatin who, after retreat- 
ment with cisplatin, developed a severe hypersensitivity reaction and died [9]. In another article, it was reported that a patient with ovarian cancer suffered anaphylactic shock and could not be resuscitated after approximately 400 micrograms of cisplatin. She had a seizure during treatment with carboplatin due to a relapse and was switched to cisplatin because she had responded well to cisplatin before [10]. For lung cancer patients, although some studies have reported hypersensitivity reactions to cisplatin or carboplatin [17] [18], we believe our patient was the first reported case of a hypersensitivity and cross-reactivity to both carboplatin and cisplatin.

Mild reactions such as pruritus, can be treated immediately with intravenous antihistamines and steroids. More severe reactions, especially those involve the cardiorespiratory system, should be treated with adrenaline $(0.5-1.0 \mathrm{ml}$ of 1:1000) for intramuscular injection. An early study evaluating cisplatin in combination with vindesine for the treatment of lung cancer reported that in patients who received six or more cycles of combination chemotherapy, five of 21 patients developed "anaphylaxis", which caused rash, pruritus, respiratory distress, and mild hypotension that appeared within 10 minutes of the cisplatin administration. In all cases, the reaction subsided when discontinuing the cisplatin infusion and administering adrenaline and diphenhydramine [18]. In our patient, life-threatening respiratory and/or circulatory problems were evident, so the administration of adrenaline is promptly and necessary.

O'Ceabhaill et al. published a retrospective study of 777 patients with recurrent ovarian cancer retreated with carboplatin. The result showed that, compared to the standard 30-minute schedule, an incrementally increasing, 3-hour infusion was associated with a lower rate of hypersensitivity to carboplatin [19]. Patients may also be re-submitted to infusion reduction and premedication or follow a desensitization protocol [20]. Skin testing helps eliminate the possibility of cross-reaction between platinum compounds and makes continued effective platinum-based chemotherapy safe. However, our hospital did not perform skin tests and therefore, in general, a desensitization protocol would not be warranted in the setting of chemotherapy for metastatic or recurrent lung cancer patients with severe anaphylactic reaction. Hence, discontinuation of platinum chemotherapy entirely appears to be the most reasonable decision.

\section{Conclusion}

To our knowledge, this is the first case of hypersensitivity and cross-reactivity to both carboplatin and cisplatin in NSCLC patients. The amount of previous platinum treatments and the total exposure duration are associated with the possibility of hypersensitivity. Retreatment with cisplatin for relapsed patients having carboplatin-allergy cannot be recommended. The decision is made with careful consideration of the potential risks and benefits. Accordingly, doctors and nurses should be cautious about the possible platinum hypersensitivity in the lung cancer patients and be ready and capable to respond to this emergency. 


\section{Acknowledgements}

The authors would like to thank Dr. Nguyen Thanh Hang-Department of Scientific Research and International Cooperation Department, Hanoi Oncology Hospital for editing English and providing consultation on academic writing in this manuscript.

\section{Conflicts of Interest}

The authors declare no conflicts of interest.

\section{References}

[1] Sung, H., Ferlay, J., Siegel, R.L., Laversanne, M., Soerjomataram, I., Jemal, A., et al. (2021) Global Cancer Statistics 2020: GLOBOCAN Estimates of Incidence and Mortality Worldwide for 36 Cancers in 185 Countries. CA: A Cancer Journal for Clinicians, 71, 209-249. https://doi.org/10.3322/caac.21660

[2] Rossi, A. and Di Maio, M. (2016) Platinum-Based Chemotherapy in Advanced Non-Small-Cell Lung Cancer: Optimal Number of Treatment Cycles. Expert Review of Anticancer Therapy, 16, 653-660. https://doi.org/10.1586/14737140.2016.1170596

[3] Jamieson, E.R. and Lippard, S.J. (1999) Structure, Recognition, and Processing of Cisplatin-DNA Adducts. Chemical Reviews, 99, 2467-2498. https://doi.org/10.1021/cr980421n

[4] Makrilia, N., Syrigou, E., Kaklamanos, I., Manolopoulos, L. and Saif, M.W. (2010) Hypersensitivity Reactions Associated with Platinum Antineoplastic Agents: A Systematic Review. Metal-Based Drugs, 2010, 1-11. https://doi.org/10.1155/2010/207084

[5] Kook, H., Kim, K.M., Choi, S.H., Choi, B.S., Kim, H.J., Chung, S.Y., et al. (1998) Life-Threatening Carboplatin Hypersensitivity during Conditioning for Autologous PBSC Transplantation: Successful Rechallenge after Desensitization. Bone Marrow Transplantation, 21, 727-729. https://doi.org/10.1038/sj.bmt.1701161

[6] Sliesoraitis, S. and Chikhale, P.J. (2005) Carboplatin Hypersensitivity. International Journal of Gynecological Cancer, 15, 13-18. https://doi.org/10.1136/ijgc-00009577-200501000-00003

[7] Koren, C., Yerushalmi, R., Katz, A., Malik, H., Sulkes, A. and Fenig, E. (2002) Hypersensitivity Reaction to Cisplatin during Chemoradiation Therapy for Gynecologic Malignancy. American Journal of Clinical Oncology, 25, 625-626.

https://doi.org/10.1097/00000421-200212000-00020

[8] Markman, M., Kennedy, A., Webster, K., Elson, P., Peterson, G., Kulp, B., et al. (1999) Clinical Features of Hypersensitivity Reactions to Carboplatin. Journal of Clinical Oncology, 17, 1141. https://doi.org/10.1200/JCO.1999.17.4.1141

[9] Dizon, D.S., Sabbatini, P.J., Aghajanian, C., Hensley, M.L. and Spriggs, D.R. (2002) Analysis of Patients with Epithelial Ovarian Cancer or Fallopian Tube Carcinoma Retreated with Cisplatin after the Development of a Carboplatin Allergy. Gynecologic Oncology, 84, 378-382. https://doi.org/10.1006/gyno.2001.6519

[10] Zweizig, S., Roman, L.D. and Muderspach, L.I. (1994) Death from Anaphylaxis to Cisplatin: A Case Report. Gynecologic Oncology, 53, 121-122. https://doi.org/10.1006/gyno.1994.1098

[11] Morgan, J.S., Adams, M. and Mason, M.D. (1994) Hypersensitivity Reactions to Carboplatin Given to Patients with Relapsed Ovarian Carcinoma. European Journal 
of Cancer, 30A, 1205-1206. https://doi.org/10.1016/0959-8049(94)90489-8

[12] Sugimoto, H., Iwamoto, T., Murashima, Y., Tabata, T., Sagawa, N. and Okuda, M. (2011) Risk Factors Contributing to the Development of Carboplatin-Related Delayed Hypersensitivity Reactions in Japanese Patients with Gynecologic Cancers. Cancer Chemotherapy and Pharmacology, 67, 415-419.

https://doi.org/10.1007/s00280-010-1338-5

[13] Lenz, H.J. (2007) Management and Preparedness for Infusion and Hypersensitivity Reactions. The Oncologist, 12, 601-609. https://doi.org/10.1634/theoncologist.12-5-601

[14] Gadducci, A., Tana, R., Teti, G., Zanca, G., Fanucchi, A. and Genazzani, A.R. (2008) Analysis of the Pattern of Hypersensitivity Reactions in Patients Receiving Carboplatin Retreatment for Recurrent Ovarian Cancer. International Journal of Gynecological Cancer, 18, 615-620. https://doi.org/10.1111/j.1525-1438.2007.01063.x

[15] Ottaiano, A., Tambaro, R., Greggi, S., Prato, R., Di Maio, M., Esposito, G., et al. (2003) Safety of Cisplatin after Severe Hypersensitivity Reactions to Carboplatin in Patients with Recurrent Ovarian Carcinoma. Anticancer Research, 23, 3465-3468.

[16] Syrigou, E., Makrilia, N., Vassias, A., Nikolaidis, I., Xyla, V., Manolopoulos, L., et al. (2010) Administration of Cisplatin in Three Patients with Carboplatin Hypersensitivity: Is Skin Testing Useful? Anticancer Drugs, 21, 333-338. https://doi.org/10.1097/CAD.0b013e32833418c0

[17] Randall, J.M., Bharne, A.A. and Bazhenova, L.A. (2013) Hypersensitivity Reactions to Carboplatin and Cisplatin in Non-Small Cell Lung Cancer. Journal of Thoracic Disease, 5, E53-E57.

[18] Gralla, R.J., Casper, E.S., Kelsen, D.P., Braun, D.W., Dukeman Jr., M.E., Martini, N., et al. (1981) Cisplatin and Vindesine Combination Chemotherapy for Advanced Carcinoma of the Lung: A Randomized Trial Investigating Two Dosage Schedules. Annals of Internal Medicine, 95, 414-420. https://doi.org/10.7326/0003-4819-95-4-414

[19] O'Cearbhaill, R., Zhou, Q., Iasonos, A., Hensley, M.L., Tew, W.P., Aghajanian, C., et al. (2010) The Prophylactic Conversion to an Extended Infusion Schedule and Use of Premedication to Prevent Hypersensitivity Reactions in Ovarian Cancer Patients during Carboplatin Retreatment. Gynecologic Oncology, 116, 326-331. https://doi.org/10.1016/j.ygyno.2009.10.070

[20] Hesterberg, P.E., Banerji, A., Oren, E., Penson, R.T., Krasner, C.N., Seiden, M.V., et al. (2009) Risk Stratification for Desensitization of Patients with Carboplatin Hypersensitivity: Clinical Presentation and Management. Journal of Allergy and Clinical Immunology, 123, 1262-1267. https://doi.org/10.1016/j.jaci.2009.02.042 